\title{
Étude du diagramme des phases pression-température du système PZT : Études cristallographiques, spectroscopiques et diélectriques
}

\author{
J. Rouquette, J. Haines, V. Bornand, M. Pintard et Ph. Papet \\ Laboratoire de Physico-Chimie de la Matière Condensée, UMR CNRS 5617, \\ Université Montpellier II Sciences et Techniques du Languedoc, cc 003, Place \\ Eugène Bataillon, 34095 Montpellier Cedex 5, France
}

\begin{abstract}
Résumé : L'étude de la solution solide $\mathrm{PbZr}_{(1-\mathrm{x})} \mathrm{Ti}_{\mathrm{x}} \mathrm{O}_{3}$ (pour $\mathrm{x}=0,50$ et 0,52 ) par diffraction des rayons $\mathrm{X}$ et spectroscopie Raman, en fonction de la température, a démontré l'existence de deux phases monocliniques $\left(\mathrm{M}^{\mathrm{HT}}\right.$, pour la forme haute température et $\mathrm{M}^{\mathrm{BT}}$, pour la forme basse température), confirmant ainsi les résultats publiés récemment pour la composition PZT52/48 $(\mathrm{x}=0,48)$ [1]. Par ailleurs, les spectres Raman enregistrés à hautes pressions et basses températures sur PZT52/48 ont mis en évidence (1) l'existence d'une série de transitions entre les phases quadratique $(\mathrm{Q})$, monocliniques $\left(\mathrm{M}^{\mathrm{HT}}, \mathrm{M}^{\mathrm{BT}}\right)$ ) et cubique $(\mathrm{C})$, (2) l'étendue du domaine de stabilité des phases monocliniques à hautes pressions et basses températures, ainsi que (3) la formation, à hautes pressions, d'une phase cubique caractérisée par un désordre local.
\end{abstract}

\section{INTRODUCTION}

Les matériaux céramiques $\mathrm{Pb}\left(\mathrm{Zr}_{1-\mathrm{x}} \mathrm{Ti}_{\mathrm{x}}\right) \mathrm{O}_{3}$ de la famille des pérovskites au plomb basés sur la solution solide $\mathrm{PbZrO}_{3}-\mathrm{PbTiO}_{3}$ (PZT) sont des ferroélectriques présentant des propriétés piézoélectriques remarquables, en particulier dans la zone de transition morphotropique (MPB, pour $\mathrm{x}=0,48$ ) [2]. Pendant très longtemps, la forte réponse piézoélectrique des céramiques $\mathrm{PZT}$ dans un domaine de composition proche de la MPB a été associée à la seule coexistence, dans cette zone de transition, de deux phases ferroélectriques (l'une de symétrie quadratique, riche en $\mathrm{PbTiO}_{3}$ et l'autre de symétrie rhomboédrique, riche en $\left.\mathrm{PbZrO}_{3}\right)$. La récente découverte d'une première phase monoclinique $\left(\mathrm{F}_{\mathrm{M}}{ }^{\mathrm{HT}}\right.$ de groupe d'espace $\left.\mathrm{Cm}-\mathrm{C}_{s}{ }^{3}\right)$ au niveau de la MPB [3] a permis d'apporter un élément d'explication supplémentaire. En effet, la polarisation électrique sous cette forme est contenue dans un plan monoclinique se situant entre les directions de polarisation [001] et [111] des phases quadratique et rhomboédrique, respectivement [4].

Dans le cas de la solution solide PZT52/48, et pour des températures décroissantes, une

première transition cubique paraélectrique $\left(\mathrm{P}_{\mathrm{C}}\right.$ de groupe d'espace $\left.P m \overline{3} m-O_{h}{ }^{1}\right) \leftrightarrow$ quadratique ferroélectrique $\left(\mathrm{F}_{\mathrm{T}}\right.$ de groupe d'espace $\left.P 4 m m-\mathrm{C}_{4 v}{ }^{1}\right)$ se produit à $667 \mathrm{~K}$. Par la suite, dans l'état ferroélectrique, une deuxième transition quadratique $\leftrightarrow$ monoclinique s'opère autour de la température ambiante. Enfin, une ultime transformation a été récemment mise en évidence à 210 $\mathrm{K}$ et correspondant à une seconde phase monoclinique $\left(\mathrm{F}_{\mathrm{M}}^{\mathrm{BT}}\right.$ de groupe d'espace $\left.C c-C_{s}\right)$, de paramètre de maille $c$ doublé $[5,6]$. Même si cette transformation, obtenue par rotation antiferrodistorsive des octaèdres d'oxygènes, est commune dans les systèmes pérovskites (comme $\mathrm{SrTiO}_{3}$ ) [7], elle reste néanmoins le sujet de nombreuses controverses [8,9].

Toutes ces études témoignent - si besoin en est - de la richesse et de la complexité du diagramme de phases de ces solutions solides PZT au voisinage de la MPB. Paradoxalement, peu de recherches ont été initiées pour évaluer la stabilité de ces solutions solides en fonction de la pression, malgré la sensibilité établie des propriétés diélectriques et piézoélectriques des PZT aux sollicitations et contraintes mécaniques [10]. Jusqu'à maintenant, seules quelques mesures diélectriques, réalisées à hautes températures sur PZT52/48 et limitées à des pressions de 0,8 $\mathrm{GPa}$, ont été publiées par Pisarski et al [11,12]. Plus récemment, nos propres travaux par diffraction des rayons $\mathrm{X}$ sous pression, à température ambiante, sur la solution solide PZT52/48, 
ont montré l'existence d'une transition vers une phase de symétrie cubique aux alentours de 5 $\mathrm{GPa}$ [13]. Cependant, la persistance, jusqu'à des pressions supérieures à $18 \mathrm{GPa}$, d'un spectre Raman intense - (1) interdit pour un spectre Raman de premier ordre dans le cas d'une pérovskite cubique idéale mais (2) similaire à celui observé pour les relaxeurs ferroélectriques [14-16] nous a amené à envisager la présence à hautes pressions, au sein de la matrice cubique, de nanorégions polaires. [17]. Si cette dernière conserve, à longue échelle, une structure de type

$P m \overline{3} \mathrm{~m}$, le désordre local qui la caractérise la rend donc non paraélectrique.

Dans un premier temps, et suite à l'analyse conjointe des résultats de diffraction des rayons $\mathrm{X}$ et de spectroscopie Raman en fonction de la température, pour différentes compositions PZT $(0.48 \leq \mathrm{x} \leq 0.52)$, une nouvelle ébauche du diagramme de phase au niveau de la MPB sera présentée. Par la suite, nous verrons comment l'étude par spectroscopie Raman, à basses températures et hautes pressions, pour la solution solide PZT 52/48, nous a permis de confirmer (1) l'existence de la nouvelle phase cubique "désordonnée », et (2) l'étendue des domaines de stabilité des phases monocliniques à hautes pressions et basses températures. Ce travail, de par la notion d'adaptabilité des propriétés qu'il introduit, ouvre de nouvelles perspectives d'applications pour les matériaux de la famille des PZT.

\section{PROCEDURE EXPERIMENTALE}

Les solutions solides de $\mathrm{Pb}\left(\mathrm{Zr}_{1-\mathrm{x}} \mathrm{Ti}_{\mathrm{x}}\right) \mathrm{O}_{3}$ ont été synthétisées à l'état solide par réactions conventionnelles à partir d'oxydes de très hautes pureté (>99,9\%) [1,13]. Des cartographies micro-Raman ont démontré l'excellente homogénéité des échantillons analysés, sur une surface sondée de $25,7 \times 5,2 \mu \mathrm{m}^{2}$. Les spectres de diffraction des rayons $\mathrm{X}$ ont été enregistrés sur un diffractomètre Philips X'Pert en utilisant la radiation $\mathrm{Cu} \mathrm{K}_{\alpha 1}$. Des affinements Rietveld ont été effectués avec le programme FULLPROF [18].

Les expériences Raman, à pression atmosphérique et températures variables, ont été effectuées dans un cryostat à cycle fermé d'hélium de type Oxford Instruments Microstat. Les spectres Raman ont été obtenus à l'aide d'un triple monochromateur Jobin-Yvon T64000, équipé d'un microscope Olympus et d'une caméra CCD refroidie à l'azote liquide. La température est mesurée au moyen d'une diode de silicium. L'ensemble des résultats présentés dans les études précédentes se réfêre à la radiation $514.5 \mathrm{~nm}$ de l'argon, malgré la forte absorption des matériaux de la famille PZT dans ce domaine de fréquence. Afin de limiter ces phénomènes d'absorption (chauffage local intense, vibrations de surfaces), toutes nos expériences ont été réalisées en rétrodiffusion avec la radiation de l'ion Krypton à $647.1 \mathrm{~nm}$.

Les expériences Raman, à hautes pressions et basses températures, ont été effectués en utilisant une cellule à enclumes de diamant à membrane, montée dans un cryostat à He à cycle fermé couplé à un spectromètre Jobin-Yvon U 1000 et équipé d'une CCD refroidie à l'azote liquide. Les échantillons ont été introduits avec un cristal de rubis comme témoin de pression. Ainsi, la pression induite est estimée en tenant compte du déplacement de la raie de fluorescence $R_{1}$ du rubis [19], la température [20] étant toujours mesurée avec une diode de silicium.

\section{RESULTATS}

\subsection{Diffraction des rayons $X$}

Dans les conditions normales de température et de pression, les résultats présentés dans les précédentes publications, et se référant à la diffraction des rayons $\mathrm{X}$ haute résolution 
(rayonnement synchrotron) et/ou la diffraction neutronique, prévoient une coexistence de phases pour PZT52/48. Toutefois, les conclusions sont encore divergentes quant à leur nature exacte (monoclinique et/ou quadratique et/ou rhomboédrique) [21-23].
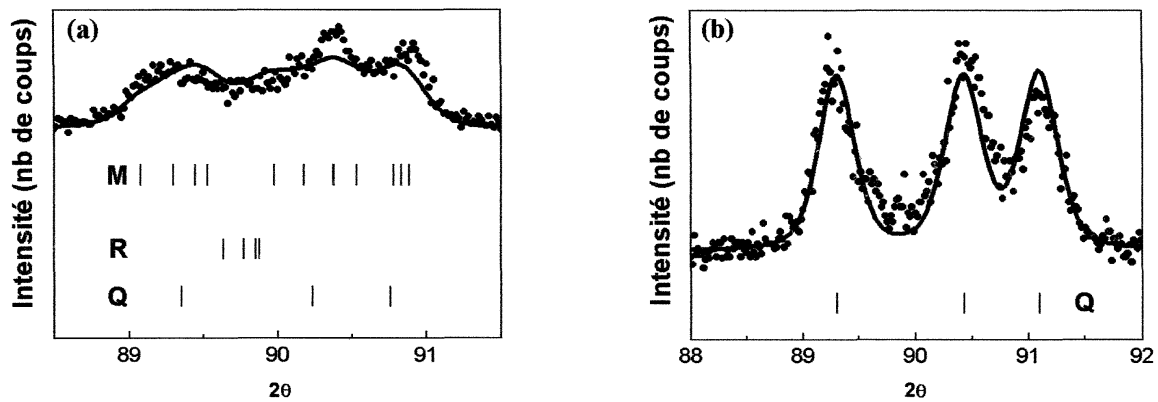

Figure 1: Détail des résultats des affinements de structure dans la région angulaire du diffractogramme correspondant à la réflexion pseudocubique (123) de PZT52/48 (a) et PZT50/50 (b) pour une symétrie monoclinique $(\mathrm{M})$, quadratique $(\mathrm{Q})$, et rhomboédrique $(\mathrm{R})$.

Dans le cas présent, les affinements Rietveld indiquent un mélange des phases monoclinique et rhomboédrique en proportion $90 \%$ et $10 \%$, respectivement. Sur la figure 1a, correspondant à la réflexion pseudo cubique (123) de l'échantillon PZT52/48, sont précisées les positions des pics de diffraction pour une structure idéale de type monoclinique, rhomboédrique ou quadratique. La figure $1 \mathrm{~b}$ correspond à la même région angulaire du diffractogramme pour l'échantillon PZT50/50. Dans ce cas, la structure quadratique, déjà reportée par diffraction synchrotron et par diffraction neutronique, est facilement affinée. Les résultats se rapportant à des compositions de plus en plus riches en Ti (PZT48/52 ...) montrent - en toute logique - que la structure reste quadratique.

\subsection{Spectroscopie Raman à température variable}

La spectroscopie Raman est un outil très puissant pour caractériser les matériaux ferropiézoélectriques. En effet, cette technique est directement reliée aux entités polaires. Ainsi, dans le cas de matériaux possédant une polarisation spontanée qui dépend de la structure, les transitions de phases sont directement observables par l'étude des phonons. Malheureusement, en l'absence de monocristaux de PZT, des échantillons nanométriques polycristallins ont été utilisés, d'où des problèmes de recouvrement de bandes rendant difficile une attribution univoque des modes. Toutefois, les résultats obtenus pour différentes compositions au voisinage de la MPB nous ont permis d'observer et d'identifier précisément les transitions de phases présentes dans ce type de matériaux.

Tous les modes de vibration de symétrie quadratique $\mathrm{E}$ doivent perdre leur dégénérescence à la transition $\mathrm{Q} \rightarrow \mathrm{M}^{\mathrm{HT}}$ pour se transformer en $\mathrm{A}^{\prime}+\mathrm{A}^{\prime \prime}$, ceux-ci étant actifs en spectroscopie Raman. Ainsi, la transition vers la phase monoclinique (HT) doit être accompagnée (1) d'un spectre devenant asymétrique et (2) d'au moins un nouveau mode de vibration. De même, la transition entre les deux phases monocliniques $\mathrm{M}^{\mathrm{HT}} \rightarrow \mathrm{M}^{\mathrm{BT}}$ s'accompagne d'une levée de dégénérescence, le doublement de maille générant plus de deux fois plus de modes vibrationnels.

Les spectres Raman de l'échantillon PZT52/48 entre 350 et $10 \mathrm{~K}$ sont reportés sur la figure 2. Ces spectres sont caractérisés par de larges pics [23] qui s'affinent (augmentation de la durée de vie des phonons) et dont l'intensité augmente lorsque la température diminue, devenant 
de plus en plus asymétriques jusqu'à se scinder. La transformation de la phase quadratique vers la phase monoclinique est identifiée par cette levée de symétrie des modes de vibrations contenus dans les domaines de fréquence $125-145 \mathrm{~cm}^{-1}$ et $230-280 \mathrm{~cm}^{-1}$ et par l'apparition d'un faible épaulement à $80 \mathrm{~cm}^{-1}$; cette transition est détectée sur les spectres entre 300 et $310 \mathrm{~K}$, en accord avec la valeur issue de mesures diélectriques sur cette composition [3,5]. Lorsque la température diminue encore, la transition entre les deux phases monocliniques s'accompagne de l'apparition de nouveaux modes localisés à 170,360 et $450 \mathrm{~cm}^{-1}$ dès $210 \mathrm{~K}$ (température de transition observée par mesures diélectriques [5]). A ce niveau, notons que ces fréquences de vibration associées au doublement de la cellule élémentaire avaient préalablement été calculées sur d'autres pérovskites au plomb, notamment $\mathrm{PbSc}_{1 / 2} \mathrm{Nb}_{1 / 2} \mathrm{O}_{3}$ et $\mathrm{PbSc}_{1 / 2} \mathrm{Ta}_{1 / 2} \mathrm{O}_{3}$ [14]. Ainsi, la présente étude établit sans ambiguité l'existence de la phase monoclinique basse température.

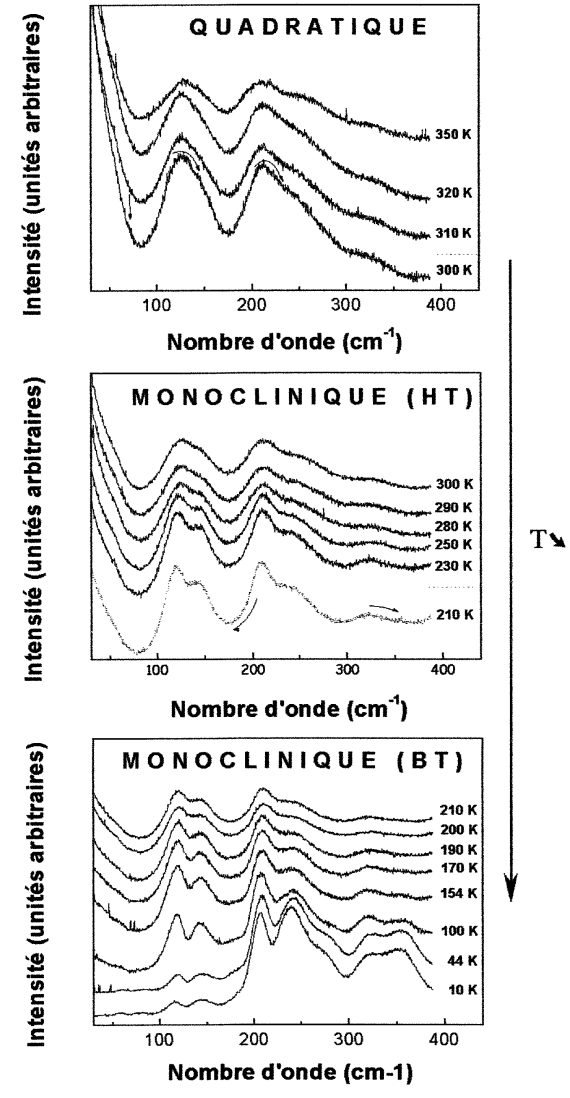

Figure 2 : Spectres Raman de PZT52/48 entre 350 et $10 \mathrm{~K}$.
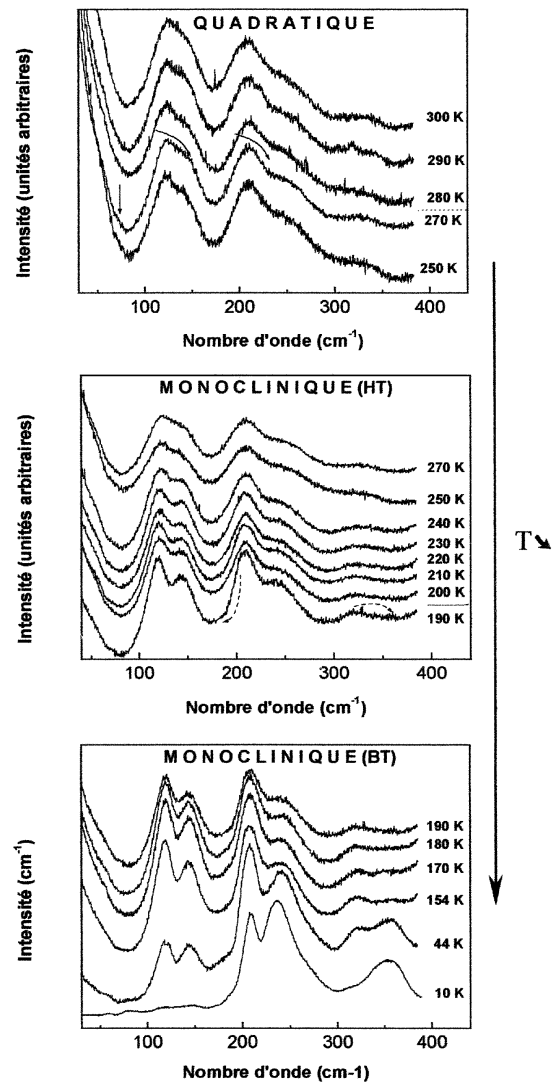

Figure 3 : Spectre Raman de PZT 50/50 entre 300 et $10 \mathrm{~K}$.

Les spectres Raman de l'échantillon PZT50/50 entre 300 et $10 \mathrm{~K}$ sont illustrés sur la figure 3. Ceux-ci présentent exactement les mêmes caractéristiques que les spectres de la composition précédente. La transition $\mathrm{Q} \rightarrow \mathrm{M}^{\mathrm{HT}}$ se produit entre 280 et $270 \mathrm{~K}$ comme en témoigne l'apparition d'un mode à $80 \mathrm{~cm}^{-1}$ et l'asymétrie progressive des pics situés à 125- 
$145 \mathrm{~cm}^{-1}$ et $230-280 \mathrm{~cm}^{-1}$. Une diminution supplémentaire de la température s'accompagne, vers $190 \mathrm{~K}$, de nouveaux modes à 170,360 et $450 \mathrm{~cm}^{-1}$ correspondant à la transition $\mathrm{M}^{\mathrm{HT}} \rightarrow \mathrm{M}^{\mathrm{BT}}$.

La figure 4 représente le diagramme de phases en fonction de la température, pour les compositions étudiées au voisinage de la MPB. Les températures de transition pour PZT48/52 ont été obtenues par une analyse similaire des spectres Raman. Comme le laisse transparaître cette nouvelle ébauche, les domaines d'existence des phases monocliniques seraient plus étendus que ce que les précédentes études laissaient envisager. Même s'il convient de confirmer les résultats observés pour chaque échantillon par des mesures complémentaires, par exemple des caractérisations diélectriques en fonction de la température, ceux-ci montrent l'extrême sensibilité de la spectroscopie Raman pour de tels matériaux.

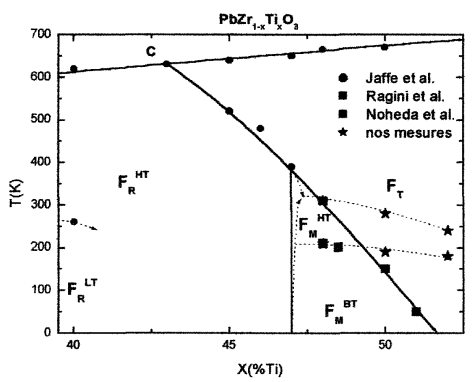

Figure 4 : Diagramme de phases pour $0,40<x<0,53$

\subsection{Spectroscopie Raman en fonction de la température et de la pression}

Les spectres Raman de l'échantillon PZT52/48 en fonction de la pression à $298 \mathrm{~K}, 154 \mathrm{~K}$ et $44 \mathrm{~K}$ sont illustrés sur la figure 5. Quelle que soit la température, une augmentation de la pression se traduit par une chute d'intensité des modes de vibration localisés dans la région $450-650 \mathrm{~cm}^{-1}$, voire même la suppression du signal situé dans la région $125-145 \mathrm{~cm}^{-1}$. Cette disparition, à $5 \mathrm{GPa}$ à température ambiante, correspond à une transition vers une phase de symétrie cubique comme le prouve les expériences diffraction des rayons $\mathrm{X}$ sous pression [13]. Ces modes disparaissent à $7,5 \mathrm{GPa}$ à $154 \mathrm{~K}$ et $9 \mathrm{GPa}$ à $44 \mathrm{~K}$.

$\mathrm{Ce}$ comportement en pression est très distinct de celui de $\mathrm{PbTiO}_{3}$ pour lequel on retrouvait une transition $Q \rightarrow C$ classique de second ordre avec les modes mous $A_{1}(1 T O)$ et $E(1 T O)$ en dessous de $150 \mathrm{~cm}^{-1}$. Dans le cas présent, le durcissement de mode constaté indique un mécanisme de transition autre que celui de type displacif évoqué pour $\mathrm{PbTiO}_{3}$ [24]. La différence de comportement entre les différents matériaux PZT et PT est d'autant plus évidente que la transition vers la phase cubique ne s'accompagne pas d'une disparition totale du signal Raman. La persistance de spectres Raman relativement intenses pour une telle structure (cubique) est la signature d'un désordre local dû à des entités polaires, provenant de déplacements locaux de cations et/ou d'anions. Par référence aux travaux portant sur les systèmes de type relaxeur, ce désordre local, responsable du non respect des règles de sélection Raman, peut se traduire par l'apparition de modes infra rouge $F_{1 u}$ et $F_{2 u}$ ou bien encore par un doublement local de la maille cubique $[14,15]$. Cette dernière hypothèse est confortée par la présence de modes à environ 80 et $360 \mathrm{~cm}^{-1}$ dans les spectres des phases monoclinique basse-température et cubique haute-pression. Ces modes sont, comme nous l'avons déjà mentionné, caractéristiques d'un doublement de maille. 


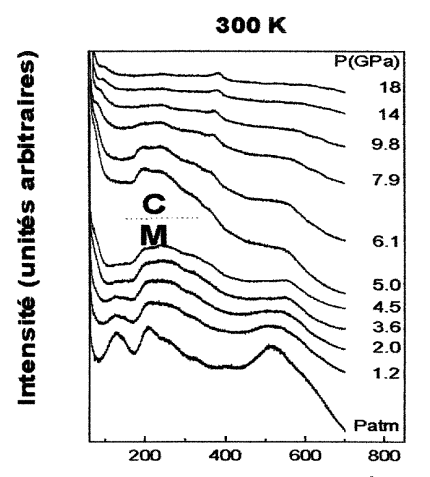

Nombre d'onde $\left(\mathrm{cm}^{-1}\right)$ $164 \mathrm{~K}$

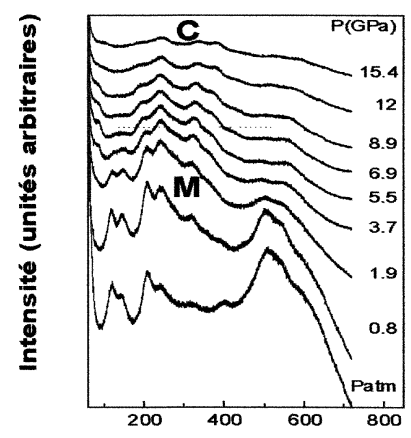

Nombre d'onde $\left(\mathrm{cm}^{-1}\right)$ $44 \mathrm{~K}$

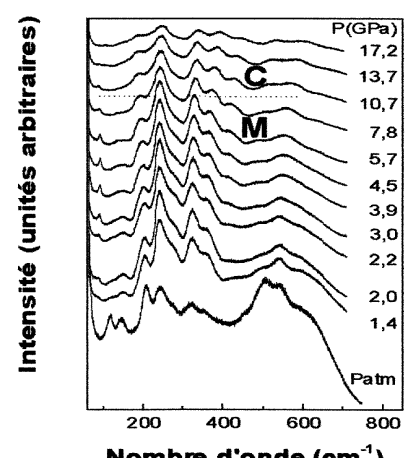

Nombre d'onde $\left(\mathrm{cm}^{-1}\right)$

Figure 5: Spectres Raman en fonction de la pression à 300,154 et $44 \mathrm{~K}$.
Un diagramme de phase pression-température (P-T) pour la composition PZT52/48 est proposé sur la figure 6. Parmi les spécifités nouvelles, on peut citer (1) l'étendue des domaines de stabilité des phases monocliniques à hautes pressions et basses températures et (2) l'existence de deux phases cubiques, l'une paraélectrique $\left(\mathrm{P}_{\mathrm{C}}\right)$, l'autre désordonnée $\left(\mathrm{F}_{\mathrm{C}}\right)$ et caractérisée par l'existence d'un spectre Raman.

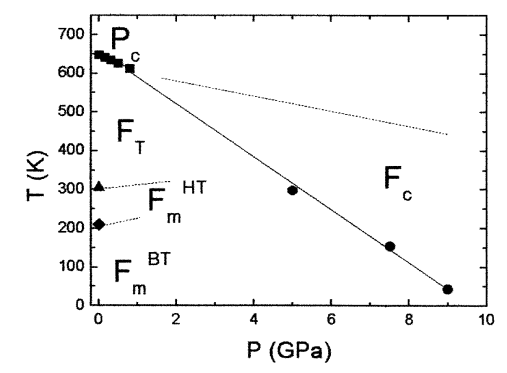

Figure 6 : Diagramme de phase $\mathrm{P}-\mathrm{T}$ de PZT52/48 $\mathrm{F}_{\mathrm{T}}$ (quadratique) $\rightarrow \mathrm{P}_{\mathrm{C}}$ (cubique paraélectrique) [11,12] $\boldsymbol{\Delta}: \mathrm{F}_{\mathrm{T}} \rightarrow \mathrm{F}_{\mathrm{M}}^{\mathrm{HT}}[20] \bullet: \mathrm{F}_{\mathrm{M}}^{\mathrm{HT}} \rightarrow \mathrm{F}_{\mathrm{M}}^{\mathrm{LT}}[5] \bullet:$ Nos mesures

\section{CONCLUSIONS}

La spectroscopie Raman à pression et température variables apparaît comme un outil d'analyse particulièrement bien adapté à l'étude des matériaux de la famille des PZT. Ainsi, associée à la diffraction des rayons X sur des échantillons de compositions voisines de la MPB, cette technique 
nous a permis d'identifier les deux phases monocliniques haute et basse température présentes dans ces systèmes. Par la suite, les expériences en fonction de la pression ont révélé (1) l'existence de deux phases cubiques (l'une paraélectrique, l'autre désordonnée localement) ainsi que (2) des domaines d'existence étendus des phases monocliniques lorsque la température diminue et/ou que la pression augmente. Des mesures diélectriques en fonction de la température et de la pression sont en cours pour valider ces résultats.

\section{Références}

1. J. Rouquette, J. Haines, V. Bornand, M. Pintard, Ph. Papet, B. Bonnet, F. Gorelli, Solid State Science, à paraìtre.

2. B. Jaffe, W. R. Cook, and H. Jaffe, Piezoelectric ceramics (Academic, London, 1971).

3. B. Noheda, D. E. Cox, G. Shirane, J. A. Gonzalo, L. E. Cross, and S.-E. Park, Appl. Phys. Lett. 74, 2059 (1999)

4. B. Noheda, Current Opinion Solid State Mater. Sci. 627 (2002).

5. Ragini, Mishra SK, Pandey D, Lemmens H, Van Tendeloo G, Phys. Rev. B 64, 054101 (2001)

6. D. M. Hatch, H. T. Stokes, R. Ranjan, Ragini, S. K. Mishra, D. Pandey, B. J. Kennedy, Phys. Rev. B 65, 212101 (2002)

7. Shirane, Yamada, -Y, Physical Review 177 (2), 858 (1969)

8. J. Frantti, S. Ivanov, S. Eriksson, M. Kakihana, Phys. Rev. B 66, 064108, (2002)

9. B. Noheda, L. Wu, Y. Zhu, Phys. Rev. B 66, 060103, (2002)

10. N.J. Ramer, S.P. Lewis, E.J. Mele, A.M. Rappe, AIP Conf. Proc. 436, 156(1998)

11. M. Pisarski, Ferroelectrics 81, 297 (1988)

12. M. Pisarski, Ferroelectrics 94,215 (1989)

13. J. Rouquette, J. Haines, V. Bornand, M. Pintard, Ph. Papet, R. Astier, J.M. Léger, F. Gorelli, Phys. Rev. B 65, 214102, (2002)

14. B. Mihailova, U. Bismayer, B. Güttler, M. Gospodinov, L. Konstantinov, J. Phys.: Condens. Matter 14, 091, (2002).

15. J. Kreisel, B. Dkhil, P. Bouvier, J. M. Kiat, Phys. Rev. B 65, 172101, (2002).

16. G. A. Samara, Phys. Rev. Lett. 77, 314, (2002)

17. H. Uwe, K. B. Lyons, H. L. Carter, P. A. Fleury, Phys. Rev. B 33, 6436, (1986)

18. J. Rodríguez-Carvajal, non publié

19. H. K. Mao, J. Xu, P.M. Bell, J. Geophys. Res. 91, 4673 (1986)

20. D. R. Ragan, R. Gustacsen, D. Schiferl, J. Appl. Phys. 72, 5539 (1992)

21. B. Noheda, L. Wu, Y. Zhu, Phys. Rev. B 66, 060103, (2002)

22. Ragini, Mishra SK, Pandey D, J. of Appl. Phys. 92, 3266 (2002)

23. J. Frantti, S. Ivanov, S. Eriksson, M. Kakihana, Phys. Rev. B 66, 064108, (1996)

24. J. A. Sanjurjo, E. López-Cruz, G.Burns, Phys. Rev. B 28, 7260, (1983) 